\title{
Predictive Maintenance Tools - A Global Survey
}

\author{
${ }^{1,2}$ Joao Rodrigues, ${ }^{3,4}$ Jose Torres Farinha, ${ }^{1}$ Antonio Marques Cardoso
}

\author{
${ }^{1}$ CISE, Universidade da Beira Interior, 6201-001, Covilh $\gamma$, PORTUGAL \\ ${ }^{2}$ Universidade Lus $\sigma$ fona, Campo Grande 376, 1749-024, Lisboa, PORTUGAL \\ ${ }^{3}$ Polytechnic Institute of Coimbra - ISEC, Quinta da Nora, 3030-199 Coimbra, PORTUGAL \\ ${ }^{4}$ CEMMPRE, Coimbra University, DEM, Polo 2, 3030-290 Coimbra, PorTUGAL.
}

\begin{abstract}
The importance given to the maintenance in the industrial world has grown over time, with new methods, new procedures and new challenges, due to the availability of new technologies. This paper focus on a global survey about predictive maintenance tools that support predictive maintenance, from the time series and decision trees until Artificial Intelligence. The approach of the several tools that can help the prediction is holistic, because new tools do not eliminate the importance of the old ones: they are complimentary and each new tool that is developed add potential for a better prediction. Additionally, it must be emphasized that some tools, that seem new are, in practice, old tools with new and powerful computational devices, assuming a new and strategic importance nowadays.
\end{abstract}

Keywords: Condition Monitoring; Predictive Maintenance; Time Series; Neural Networks; Multivariate Statistics; Artificial Intelligence

Received: June 9, 2020. Revised: November 17, 2020. Accepted: January 6, 2021. Published: January 20, 2021.

\section{IQMRGXFWRQ}

The maintenance concept has a wide range of interpretations and approaches. Nowadays, the predictive maintenance corresponds to one of the most important approaches, aiming to maximize the equipment's availability.

Some of these concepts have been referred by authors and standards through the time. Sacristan [1] refers that maintenance is based on techniques that ensure the correct use of buildings and facilities and their continuous operation.

Monchy [2] states that maintenance is the key to productivity and to product quality.

Kumar [3] is in line with the British Standards Institute (2015), defining maintenance as a combination of all the technical and associated administrative activities required to keep equipment, facilities and other physical assets in the desired operating condition or to restore them to this condition.
The European Standard EN 13306:2017 refers maintenance as "the combination of all technical, administrative and management actions, during the life cycle of a good, aimed at maintaining or restoring it to a state that can perform the required function". According to this standard, Predictive Maintenance is a condition-based maintenance carried out following a forecast derived from the analysis and evaluation of the significant degradation's parameters of the item.

Maintenance also includes the engineering decisions for optimizing capability, being this the ability to perform a specified function within a range of performance levels related to capacity, rate, quality, safety and responsiveness [4].

Some relevant studies can be found in the papers by $\mathrm{Niu} \& \mathrm{Hu}[5]$ and Ibrahim, Taha \& Shalaby [6].

In an aggressive and globalized market like today, the company has been tirelessly 
looking for new management tools to provide a competitive advantage, as is the case of predictive maintenance [7].

This paper is structured as follows:

Firstly, a general maintenance framework is made, addressing the definition of maintenance and its importance in industrial success.

Secondly, the history of maintenance is addressed, which highlights the evolution of maintenance over time, identifying the various stages that maintenance has undergone to date.

Section three presents the concept of fault diagnosis, later presenting the FMEA and FMECA analysis.

Section four addresses the predictive maintenance, identifying tools and support methods that, with the aid of artificial intelligence, can be used to benefit predictive maintenance, thereby increasing the availability of assets and decreasing their cost.

At the end, the paper presents a brief conclusion followed by thanks and the respective bibliographic references that support it.

\section{FURP $\square$ IDJQRMVIR3 UHAFWRQ}

The first generation of maintenance lasted until the middle of the $20^{\text {th }}$ century; before that date the equipment used in maintenance was simple and usually oversized for the process.

In the 1920s, industries began to understand the importance maintenance could have on their equipment [8].

After the First World War, industry was pressured to increase their production. Due to this pressure, industries formed specialized teams to repair the damage in the shortest possible time, thus preceding palliative and curative maintenance [9].

In the Second World War, industries needed to produce more than ever. The evolution of commercial aviation in the 1940s required the development of Maintenance Engineering. From the 1950s, industries began to adopt production lines and this was the birth of the second generation of maintenance.

In the 60s there was a great need for production, which meant that companies had to use two or three shifts of work. It was mandatory to avoid and foresee malfunctions in industrial equipment. Allied to this need for production, there was an evolution in computing, which allowed more robust mathematical and statistical processes to be applied.

Combining more accurate measuring devices and robust mathematical procedures it began to be possible to predict future breakdowns, appearing for the first time the predicted maintenance or condition maintenance.

In the 70s a new broader concept of maintenance was born in Europe, whose name was Terotechnology [10].

\section{Fault Diagnosis}

According to European Standard EN 13306:2017, a failure is the loss of the ability of an item to perform a required function after failure of the item has a fault, which may be complete or partial.

There are many tools to aid diagnosis, since the classical FMEA until Expert Systems for fault diagnosis.

These tools are strategic, not only to identify the root causes but, usually, they help to solve the fault, through specific procedures that guide the technicians. There are a lot of work about this subject in all technological fields. Next will be presented some references, only to signal the importance of this subject.

Yang [11], in 2004, implemented a system that integrates Case-Based Reasoning (CBR) with Petri Nets for the fault diagnosis of induction motors; from the CBR system the technicians retrieve the information from previous cases which closely resemble and solve the new problem using the information from the previous cases. They present an approach that integrates Case-Based 
Reasoning with an Adaptive Resonance Theory (ART) and a Kohonen Neural Network (KNN) - ART-KNN - to enhance fault diagnosis; when solving a new problem, the Neural Network is used to make hypotheses and to guide the CBR module to search for a similar previous case that supports one of the hypotheses.

Yin \& Zhao [12], in 2016, propose a mathematical model to formulate a fault diagnosis problem in High-Speed Railways, involving the definition of fault evidence vectors and reason vectors through the analyze of data collected in WuhanGuangzhou high speed railway; a Deep Belief Network and its training procedures are developed on the basis of Restricted Boltzmann Machine; the authors also propose a diagnosis network trained and validated with real-world data, comparing a Deep Belief Network based fault diagnosis network with a K-Nearest Neighbor and Artificial Neural Network with Back Propagation.

The Augmented Reality is another complimentary tool to aid minimizing the intervention time to correct a fault. For example, Ghimire, Krishna \& Luh [13] in 2016 presented an integrated Augmented Reality based framework to monitor an HVAC system (or any cyber-physical system) in real-time, which permits to access the system information remotely and, based on this, to prevent or to minimize the system downtime.

\subsection{FMEA \& FMECA}

Criticality analysis is based on failure mode evaluation analysis. Criticality means that the failure probability of the equipment is very high. The minor failure of critical equipment may lead to a severe impact on the performance of that equipment. So, critical equipment needs a higher degree of maintenance activity and maintenance frequency to prevent any failure [14].

Among the variables that allow the evaluation of equipment's criticality; the safety factor represents the associated risk in case of failure. If the probability of injury (man or machine) is high, in the case of equipment failure, this value is given by the safety factor and the lower the chances of injury - the lower is the safety factor's value [14].

Facing a systematic analysis approach, Failure Mode and Effects Analysis (FMEA) and, Failure Mode Effects and Criticality Analysis (FMECA), allow the identification of existing problems in the process, that may arise, given the examination of previous errors, through a hierarchy of potential failures, and making recommendations to avoid them through maintenance techniques.

\subsection{Fault Trees}

Through the Fault Trees (FT) tool it is possible to identify the main causes that determine the occurrence of undesirable events from the logical combination of the failures of the various components of the system itself.

The Fault Tree technique appeared in 1962 and was created by Watson at Bell Telephone Laboratories. The failing tree emerged with the aim of evaluating the security control system of the Minuteman ballistic missile.

In 1965, this same technique was improved by Watson and Haasl for Boeing Company in their aeronautical projects.

In this way, applying the FT in industrial maintenance can benefit it because it allows the identification of potential failures, and a much more accurate diagnosis of asset failures as well as prediction.

Milde \& Hotz [15], in 2000, presented a system that generates FT based on models of the technical devices: the FT is generated automatically based on the device model. Since the process of creating a FT is very fast, the cost of providing, modifying and maintaining diagnostic equipment can be drastically reduced. 


\subsection{Dynamic modelling}

\subsubsection{Petri Nets}

Petri Nets (PN) were born from Carl Adam Petri's dissertation in 1962, and this same research was presented at the Faculty of Mathematics and Physics at the Technical University of Darmstadt in West, Germany. The scientist Petri worked at the University of Bonn while elaborating his dissertation, with the Petri nets coming to have their first applications in the following decade [16].

Petri Nets are a mathematical and graphical modelling tool that can be applied to industrial maintenance. PN are a graphical tool, that can be used as a visual communication aid similar to block diagrams. When used as a mathematical tool, it is possible to configure the state, algebraic equations and other mathematical models that manage the behaviour of the systems under analysis [17].

A Petri Net consists of a directed, weighted and bipartite graph, composed of two structural elements: places and transitions. The place is represented graphically by a circle and the transition by a bar. Structural elements are used to create the model, in which oriented arcs connect places to transitions and transitions to places. These bows can be labelled with a positive integer, indicating their weight. An arc of weight $k$ can be interpreted as $k$ parallel arcs. Petri nets can be applied to almost any area that can be described using flow charts. Although they can be applied to many areas; the main problem with Petri Nets is their complexity: the models based on PN tend to be too large for analysis, even when addressing smaller systems. However, one should pay attention to the model's generosity, since the more general the model, the less amenable it is to analyze [17].

To apply PN to different systems, it is often necessary to add restrictions to it. Murata states that the most promising areas in terms of applicability of petri nets are performance evaluation, analysis of software systems, industrial manufacturing and control systems, logical programs, and fault-tolerant systems.

Simeu-Abazi \& Sassine [18] presented a modular modelling approach, based on the cellular decomposition of the system, using stochastic Petri Nets and Markov Chains adopting various maintenance strategies in complex production workshops, with the objective of studying its influence on the reliability and performance of the system.

Leigh \& Dunnett [19] presented a simulation model for the maintenance process of a wind turbine in order to develop a procedure that can be used to optimize the process. In this project, PN were used to simulate each type of maintenance as well as climatic conditions. The same authors even claim that Petri Nets are a good tool to model the maintenance process due to its dynamic modelling and adaptability and its ability to test optimization techniques

\subsection{Fault Diagnosis through AI}

The managers' objective is to maximize the organizations' profits, with maintenance having a key role for this. It is necessary an approach that gives awareness to the top management about the need to be involved in the process of improving the maintenance function [20]. This approach may contain a maintenance performance management system, which also acts as a rapid performance alert system [21].

Cardoso state that the term used for maintenance Key Performance Indicators (KPI) in an Industry implies that its definition should provide the following information: name, type and, finally, mathematical formula of the indicator with the necessary data for its calculation [22]. The KPI should be aligned with defined roles and responsibilities for the maintenance function against the assets for which they apply, because the right indicators can give information about opportunities for improvement and solutions for machine's problems [23]. 
Knowing the importance of measuring maintenance performance, it should be noted that measuring ought to be done since an initial state. However, even if the tools and mathematical techniques developed have not yet been applied to the maintenance area, they can still help in decision making. This management support tool, and the technical growth combined with technology will allow a much more efficient maintenance management [24]. The adoption of complex maintenance measurement systems may not be beneficial for the company, as they are more difficult to install and interpret by managers. Proof of this is the increasing tendency of the industries to use simpler models, but always keeping in mind the maintenance policy of each organization [3].

\section{PUHAFWMHO DOQMDDQFH}

The classical tools for predictive maintenance are based on time series, which provides interesting algorithms to make prediction.

\subsection{Time Series}

A time series is simply a series of observations recorded sequentially over time [25], [26]. They are applied in several areas, engineering is no exception, and they can be used in the field of prediction of industrial maintenance [27].

Box emphasizes the analysis of time series, which are based on the application of methodologies and the integrated and Autoregressive Moving Average Models (ARMA), as well as extensions of these models.

The analysis of time series aims to understand the dependence of current and past data and exploit them; so, it is possible to predict the future behavior [28]. For example, in the Control of Industrial Processes, the forecast methods are essential to characterize products, to understand the quality of the processes and, thus, to adjust the processes in order to avoid over production capacity [29]. In the general economy, various institutions, such as governments, financial institutions and political organizations make decisions based on forecasts of economic variables, such as Gross Domestic Product, the unemployment rate or inflation, for exports and imports [30].

\subsubsection{Time Series traditional tools}

A stochastic process can be defined as a finite or infinite sequence of random variables and a time series can be understood as one of the infinite realizations of a stochastic process [31].

In most time series techniques, it is assumed that data is stationary, so, given this characteristic, it is important to know that a stationary process is a process in which the mean, the variance and the autocorrelation structure do not change over time.

Auto Regressive Moving Average (ARMA) provides a parsimonious description of a stationary stochastic process in terms of two polynomials, one for the Auto Regression (AR) and the second for the Moving Average (MA).

Autoregressive Integrated Moving Average (ARIMA) is a generalization of the autoregressive model of moving averages (ARMA). Both models are adjusted to the time series data for a better understanding of the data or to predict future points in the series. ARIMA models are applied in some cases where the data show evidence of no stationarity, in which an initial differentiation step (corresponding to the "integrated" part of the model) can be applied one or more times to eliminate the non-stationarity [25].

The main disadvantage of the ARIMA model is that it assumes a linear relationship between the dependent and independent variables, which is not always the case [32]. Multivariate Time Series (MTS) is one of the most commonly used methods for Multivariate Time Series (MTS); the forecasting is done through the Vector Auto Regression (VAR). The MTS models are 
designed to capture the dynamic of multiple time series simultaneously and leverage dependencies across these series for more reliable predictions [33].

\subsubsection{Bootstrap}

The bootstrap has already been applied to the conservative estimate of reliability with samples [34]. This method of random sampling is a very valuable tool when there is a database with little data. The bootstrap technique tries to accomplish what would be desirable to carry out in practice, if this is possible [35].

\subsection{Artificial Intelligence}

The modern study of intelligence is based on the work of Charles Spearman, who proposed that it ought to be understood as a general skill that brought together as a set of intellectual tasks with specific skills [36].

John McCarthy defined Artificial Intelligence as "the science and engineering of making intelligent machines, especially smart computer programs". Artificial Intelligence has been widely used in recent years for essentially three reasons: big data; cloud computing; and the latest machine learning [37].

Robert Sternberg states that "intelligence is the ability to learn from experience and to adapt, shape, and select environments" [38]. Acting rationally, a machine with Artificial Intelligence must be programmed to learn and solve manifold problems, whether simple or complex. It should also be noted that for a machine to be designated as an intelligent machine must pass the Turing test. The name of the test is due to the computer scientist and mathematician Alan Turing, given that he made great contributions to the development of computing [39].

Before the introduction of the concept of Artificial Intelligence, there was a gestation period between 1943 and 1955. In this timeframe, a model was developed for the first artificial neuron created by McCulloch and Pitts, and then, Minsky created the first Artificial Neural Network that had 40 neurons. In 1955, it was proposed that a group of 10 carefully selected scientists work in the field of Artificial Intelligence for two months, in the summer of 1956, at Dartmouth College in Hanover, New Hampshire.

The authors of this proposal believed that it would be possible, during that summer, to reach a significant advance in the area of Artificial Intelligence. In the same proposal, the authors listed problems of Artificial Intelligence, namely: Automatic Computers; How Can a Computer be Programmed to Use a Language; Neuron Nets; Theory of the Size of a Calculation; Self-promotion, Abstractions, Randomness and Creativity. And, then, Artificial Intelligence was born. In the 1950s and 1960s, there was a great deal of initial enthusiasm for artificial intelligence, as great expectations were placed on it. Despite the great enthusiasm, little has been achieved: however, the introduction of GPS, by Newell in 1959, stands out as an important step.

In the following decade, the first knowledge systems began to appear, and it was from 1980 that artificial intelligence entered industries with numerous applications of great success, saving human labor, increasing production and reducing financial costs. In the same decade, several techniques were developed, such as data mining and Machine Learning.

Artificial Intelligence had a historic milestone when an IBM chess computer called Deep Blue defeated former world chess champion Gary Kasparov. This game took place in 1997 and was released around the world.

More recently, deep learning had advances in artificial intelligence being introduced by Ian Goodfellow in 2012. In Machine Learning, systems are programmed to learn how to perform a task or several tasks; these systems learn according to the environment or historical data, while Deep Learning is a 
subcategory of Machine Learning that has vast neural networks trained by data history to perform a specific task.

\subsubsection{Markov Chains}

Markov Chains model is based on the probability distribution of state transitions generated by a transition matrix of this chain. This model can also find out the chances of the transitions occurring in several other steps (M). Given the transition matrix, the input value of the matrix obtained can be calculated by raising this same transition to the power of $\mathrm{M}$.

Markov chains enable an innovation of an efficient solution algorithm and in obtaining analytical results. In this way, it is possible to create a numerical example that will be used to conduct proposed policies of integrated statistical control and preventive maintenance. It also allows a sensitivity analysis that is conducted to analyze the impact of these same parameters of the model on ideal policies and also interrelationship between statistical control of the process itself and maintenance actions. These results can also highlight the costs that may arise from the policies proposed by the model. A Markov Process is able to explain the random behavior during its own degradation, allowing a more real approximation of the result between estimation and observation. A Markov model is a reliable model that allows to model the deterioration processes of machines and equipment, through a proposal based on the technical description of the components, thus leading to a timedependent solution.

\subsubsection{Hidden Markov Chains}

A Hidden Markov Model (HMM) is a probabilistic system designed to model a sequence as a result of a Markovian process that cannot be observed [40], [41]. The HMM algorithms are suitable for maintaining predictive control of Diesel engine conditions, because it is very robust at handling the probabilities of state condition associated to certain spectra of emissions. The implementation's success depends on the classification criteria and monitoring frequency [42]. The paper [43], entitled "Applications of predictive maintenance techniques in industrial systems", showed the possibility of applying the HMM approach to increase the reliability of a system and the performance of industrial maintenance [43].

\subsubsection{Decision Trees}

To understand how the random forest works, it is necessary to first understand how the decision trees work, because they are the fundamental components of the random forest, which is a machine learning algorithm [44].

The decision tree is a nonparametric and nonlinear supervised learning algorithm that can be used for classification and regression; the purpose is to create a model that predicts the value of an output variable depending on the inferred decision rules[44].

A decision tree is a directed acyclic graph in which each node, except the root, has exactly one parent and the root has no parents, and it is at the root where the decision process begins [45].

An example of the application of decision trees was the classification of objects for a robot manipulator. Among the existing pruning methods, the following stands out: Cost Complexity Pruning; Reduced Error Pruning; Minimum Error Pruning; Pessimistic Pruning; Error-Based Pruning; Minimum Description Length Pruning; Minimum Message Length Pruning; Critical Value Pruning [46].

Principal Component Analysis evaluates the random part of the data. There are several classification algorithms that use the decision tree, such as: ID3 [47]; CART Classification and Regression Trees [48]; C4.5 [49]; or CHAID (Chi Square Automatic Interaction Detection). It is not possible to assess which is the best 
algorithm, as the performance of the algorithms depends on the type of problem. In this research field of decision trees, the authors Breiman and Quinlan stand out.

\subsubsection{Random Forest}

The Breiman's work about random forest was decisively influenced by the early search of Amit and Geman, in 1997, on geometric feature selection: the random subspace method of Ho, in 1998, and the random split selection approach of Dietterich, in 2000 [50], [51]. Breiman throughout his various papers has shown that decision trees can produce substantial gains in accuracy when used together in regression and classification tasks; these two types of tasks represent a large percentage of machine learning systems [52].

In a regular decision tree, a single decision tree is built; however, in a random forest, many decision trees are built and the number of trees is usually defined by the user [53].

In a joint process, each tree casts a unit vote and each decision tree is used to choose the final class. In random forests, a sample of substituted data is used to build a decision tree along with the subset of variables. The sampling and subset are recorded randomly [53], [54].

\subsubsection{Artificial Neural Networks}

The human brain is a highly powerful machine capable of processing a large amount of data in a short time. It structures and organize neurons, in order to perform various calculations, with neurons being responsible for processing the data. Hawking states that "the brain is a highly complex, non-linear and parallel computer." The first conclusions were drawn at the beginning, trying to create a model similar to the human brain; the conventional computers work completely different from the human brain.

Inspired by learning from human experience, several researchers tried to create intelligent systems capable of performing tasks such as classification, image processing, and pattern recognition; the first results in the artificial neuron model and, later, a system with several interconnected neurons, were called Neural Network (NN). After the pioneering work of McCulloch and Pitts, in 1943, computer software was developed to execute algorithms such as: Stuttgart Neural Network Simulator (SNNS), the MathWorks MATLAB neural network toolbox, and the General Neural Simulation System (GENESIS). This software provides to the user a flexible environment with extensive libraries that allow to cover several applications; all this software is based on the Von Newman machine.

Recently, Artificial Neural Networks (ANN) have been used frequently in pattern recognition, classification and prediction. Artificial Neural Networks are part of Machine Learning and can complement and confront, in many cases, conventional regression methods and even multivariate statistical models [55], [56].

Artificial Neural Networks, combined with methods such as, time series, Markov chains, and multivariate statistics, can help industrial maintenance to take a step forward in predictive maintenance, increasing levels of rigor achieved. It should be emphasized that the data must be previously studied in order to choose the most suitable methods for each prediction situation [57]. The processing capacity of the network is stored in the strengths of the unit connection, obtained through a process of adapting or learning a set of training patterns.

A Neural Network is a massively parallel distributed processor, consisting of simple processing units that have a natural propensity to store experiential knowledge and hold it accountable for use.

The Neural Networks can be evaluated taking into account several factors such as, for example: latency; convergence; performance; fault tolerance; convergence data analysis; precision or, even, processing speed [58]. According to Lippmann, "the 
great potential of Neural Networks remains in the highspeed processing that could be provided through massively parallel implementation" [59].

A NN can be characterized by four properties: network topology; advanced procedure; training or learning procedure; and post training procedure [60].

In order to improve Artificial Neural Networks, in the future there will be key challenges to develop things such as: improving the design of robust models; improving the transparency of the model and allowing the extraction of knowledge from already trained networks; improving the capacity of extrapolation; and, finally, to create approaches to work with uncertainty. Neural Networks, due to their excellent selflearning properties, adaptability, fault tolerance and non-linearity, can, and should be, used to solve problems in many different areas, like agriculture, medical science, education, finance, security, logistics, management and, of course, engineering. Rahmanifard \& Plaksina [61], in 2019, show that Artificial Neural Networks can be a great help in solving optimization problems in the oil exploration and production industry. These authors consider that, "due to the frequency of use of Artificial Neural Networks in the environment of organizations, some applications of Neural Networks are already considered usual". Knowing the importance presented in this paper, a new technique to determine engine failures and conditions from lubricant samples was developed, supported on Artificial Neural Networks, "based on the measurement of the dielectric constant and the loss factor of engine lubricating oil samples". Artificial Neural Networks were used to monitor the correlation between predicted and measured dielectric characteristics; it should be noted that the Quasi-Newton backpropagation algorithm was the one that had closest results to the measurement [62].
Hongxiang, Yuntao \& Xiangjun [63], in 2011, presented a paper in which they state that Artificial Neural Networks can analyze spectroscopy data from a mining oil, revealing the type of oil, and distinguishing the routine condition of the Diesel engine from the operating condition.

Rodrigues et al. [57], in 2019, shows that Artificial Neural Networks can be used to know the condition of Diesel engine oil. The analyzed databases contain twenty-one variables, and, at the end, the authors conclude that this method can learn and make almost the same decisions as the human specialists, so, the process can be automated. Artificial Neural Networks is a strong technology, but if it is combined with the correct analysis of Big Data, it will certainly be one of the most powerful technological tools in the coming years.

\subsubsection{Support Vector Machine}

Support Vector Machine (SVM) is a supervised machine learning algorithm developed by Vapnik. This algorithm can be used for classification or regression tasks, which are really necessary in the field of condition monitoring.

The objective of SVM is to produce a classifier that works properly with unknown examples, which were not applied during training, but acquiring the ability to predict the outputs of future new entries. SVM is a very powerful classification algorithm that can be used in conjunction with the Random Forest algorithm, previously mentioned, thus providing an extremely high precision in case of predictive maintenance situations. The SVM was proposed to provide a good performance in task classification; however, if the result of the SVM's classification itself is often depleted, that means it is far from theoretically expected, due to the fact that its execution is based on algorithms for approximation of high complexity in space and time. Just as Random Forest can assist SVM, Kim [64] propose to run the SVM package with the bagging tool, which trains 
each individual SVM and the training samples are chosen randomly using a bootstrap technique - both techniques were mentioned above in this paper.

Another proposal by the same author is the reinforcement, where each individual SVM is trained using the training samples chosen according to the sample's probability distribution, which is updated proportionally to the sample error. In both methods, each SVM individual training is aggregated to make a collective decision in several ways, such as majority voting, weighting based on estimation of minimum squares and hierarchical double layer combination. After analyzing the results, it can be concluded that both training methods surpass the single SVM at the level of classification accuracy [64].

Due to the reduction of maintenance costs and, in order to increase greater productivity, equipment availability, fault detection and diagnosis, this is an extremely important research field in condition maintenance. Due to these reasons Salahshoor [65], in 2010, developed a fault detection and diagnosis model for an industrial steam turbine using a data fusion methodology. This model consisted on the fusion of an SVM classifier with an ANFIS classifier, this being the acronym for Adaptive Neuro-Fuzzy Inference System.

It is noteworthy that SVM works poorly in sets with a large volume of data, where is required a matrix inversion. Another disadvantage of the SVMs is the malfunction in the case of data with a large amount of noise; although, the SVM, in the case of outliers, always seeks for the best possible form of classification, even disregarding the existing outliers.

\section{Conclusions}

This paper presented a review on predictive maintenance tools.

Among the many tools referred along the paper, it was emphasized the importance of the Artificial Intelligence.
It was noted that, for Artificial Intelligence to produce reliable information, it is imperative that it must be based on robust databases, which often does not happen; in these databases the bootstrap can be a strategic tool in improving it.

This paper also showed how FMEA and FMECA analysis can be a mainstay in maintenance because through they it is possible to point and scale the level of gravity and frequency of all the potential failures of an equipment.

Artificial Intelligence tools can also be used to assist management decision making, and, even, to support the maintenance management.

The paper also refers the importance of the Key Performance Indicators, that help to know the maintenance status, and which equipment need more attention.

\section{Acknowledgments}

This work was supported by the European Regional Development Fund (ERDF) through the Operational Program for Competitiveness and Internationalization (COMPETE 2020), under Project POCI010145-FEDER-029494, and by National Funds through the FCT - Portuguese Foundation for Science and Technology, under Projects PTDC/EEIEEE/29494/2017, UIDB/04131/2020 and UID-Multi044632019 - DREAMS

\section{References}

[1] F. R. Sacristan, "Gestão Industrial: Manutenção Mecânica e Elétrica na Indústria e nas Oficinas". 1975.

[2] F. Monchy, "A Função Manutenção". 1989.

[3] U. Kumar, D. Galar, A. Parida, C. Stenström, and L. Berges, "Maintenance performance metrics: A state-of-the-art review," J. Qual. Maint. Eng., vol. 19, no. 3, pp. 233277, 2013, doi: 10.1108/JQME-052013-0029. 
[4] A. H. C. Tsang, "Strategic dimensions of maintenance management," $J$. Qual. Maint. Eng., vol. 8, no. 1, pp. 739, Mar. 2002, doi: $10.1108 / 13552510210420577$.

[5] G. C. Niu and Z. Hu, "Evaluation and health status prediction method of beer filling production line based on data mining technology," Int. J. Circuits, Syst. Signal Process., vol. 13, pp. 306311, 2019.

[6] R. E. Ibrahim, L. G. Taha, and A. Shalaby, "Urban expansion and pattern analysis using Shannon's entropy approach in Elminya Governorate, Upper Egypt," Int. J. Circuits, Syst. Signal Process., vol. 13, no. November, pp. 637-646, 2019.

[7] A. K. J. Nascif, "Manutenção - Função Estratégica", Qualitymar. 2007.

[8] John Moubray, "Reliability-Centered Maintenance", Second Edi. New York: Industrial Press Inc., 1997.

[9] J. T. Farinha, "A Terologia e as Novas Ferramentas de Gestão", Monitor. Portugal. 2011.

[10] T. M. Husband, "Maintenance Management and Terotechnology". 1988.

[11] B.-S. Yang, T. Han, and Y.-S. Kim, "Integration of ART-Kohonen neural network and case-based reasoning for intelligent fault diagnosis," Expert Syst. Appl., vol. 26, no. 3, pp. 387395, Apr. 2004, doi: 10.1016/j.eswa.2003.09.009.

[12] J. Yin and W. Zhao, "Fault diagnosis network design for vehicle on-board equipments of high-speed railway: A deep learning approach," Eng. Appl. Artif. Intell., vol. 56, pp. 250-259, Nov. 2016, doi: 10.1016/j.engappai.2016.10.002.

[13] R. Ghimire, K. R. Pattipati, and P. B. Luh, "Fault diagnosis and augmented reality-based troubleshooting of HVAC systems," in 2016 IEEE AUTOTESTCON, Sep. 2016, pp. 110, doi: 10.1109/AUTEST.2016.7589590.
[14] M. Niraj and P. Kumar, "FMECA Analysis (A Heuristic Approach) For Frequency of Maintenance and Type of Maintenance," Int. J. Sci. Eng. Res., vol. 3, no. 1, pp. 1-5, 2012.

[15] H. Milde and L. Hotz, "Facing Diagnosis Reality - Model-Based Fault Tree," 2000.

[16] C. Petri, "Kommunikation mit atomation." 1962.

[17] T. Murata, "Petri nets: Properties, analysis and applications," Proc. IEEE, vol. 77, no. 4, pp. 541-580, Apr. 1989, doi: 10.1109/5.24143.

[18] Z. Simeu-Abazi and C. Sassine, "Maintenance integration in manufacturing systems by using stochastic Petri nets," Int. J. Prod. Res., vol. 37, no. 17, pp. 3927-3940, Nov. 1999, doi: 10.1080/002075499189844.

[19] J. M. Leigh and S. J. Dunnett, "Use of Petri Nets to Model the Maintenance of Wind Turbines," Qual. Reliab. Eng. Int., vol. 32, no. 1, pp. 167-180, Feb. 2016, doi: 10.1002/qre.1737.

[20] M. Aoudia, O. Belmokhtar, and G. Zwingelstein, "Economic impact of maintenance management ineffectiveness of an oil and gas company," J. Qual. Maint. Eng., vol. 14, no. 3, pp. 237-261, Aug. 2008, doi: $10.1108 / 13552510810899454$.

[21] A. Parida and U. Kumar, "Maintenance performance measurement (MPM): Issues and challenges," J. Qual. Maint. Eng., vol. 12, no. 3, pp. 239-251, 2006, doi: $10.1108 / 13552510610685084$.

[22] A. J. M. Cardoso and C. P. Cabrita, "Concepts and definitions of failure and breakdown in the Portuguese maintenance standards NP EN 13306: 2007 and NP EN 15341: 2009," in 17 Ibero-American Congress on Maintenance, 2013.

[23] T. Wierman, "Developing Performance Indicators for Managing Maintenance". New York, 2005.

[24] A. Parida and G. Chattopadhyay, 
"Development of a multi-criteria hierarchical framework for maintenance performance measurement (MPM)," J. Qual. Maint. Eng., vol. 13, no. 3, pp. 241258, Aug. 2007, doi: $10.1108 / 13552510710780276$.

[25] G. E. P. Box, G. M. Jenkins, G. C. Reinsel, and G. M. Ljung, "Time Series Analysis - Forecasting and Control". 1994.

[26] C. Chatfield and H. Xing, "The Analysis of Time Series: An introduction with R", vol. 53, no. 9. 2019.

[27] G. E. P. Box, G. M. Jenkins, G. C. Reinsel, and G. M. Ljung, "Time Series Analysis Forecasting and Control", John Wiley. New Jersey, 2016.

[28] Rob J. Hyndman and Yeasmin Khandakar, "Automatic Time Series Forecasting: The forecast Package for R," J. Stat. Softw., vol. 27, no. 3, p. 22, 2008, [Online]. Available: http://www.jstatsoft.org/v27/i03/pape $r$.

[29] W. W. C. Chung and S. W. F. Leung, "Collaborative planning, forecasting and replenishment: a case study in copper clad laminate industry," Prod. Plan. Control, vol. 16, no. 6, pp. 563574, Sep. 2005, doi: 10.1080/09537280500112488.

[30] C. Granger and P. Newbold, "Forecasting Economic Time Series". New York, 1986.

[31] P. J. Brockwell and R. A. Davis, "Introduction to Time Series and Forecasting". New York: Springer, 2002.

[32] G. P. Zhang, "Time series forecasting using a hybrid ARIMA and neural network model," Neurocomputing, vol. 50, pp. 159-175, Jan. 2003, doi: 10.1016/S0925-2312(01)00702-0.

[33] Y. Bao, Z. Chen, S. Wei, Y. Xu, Z. Tang, and H. Li, "The State of the Art of Data Science and Engineering in Structural Health Monitoring,"
Engineering, vol. 5, no. 2. pp. 234242, 2019, doi: 10.1016/j.eng.2018.11.027.

[34] V. Picheny, N. H. Kim, and R. T. Haftka, "Application of bootstrap method in conservative estimation of reliability with limited samples," Struct. Multidiscip. Optim., vol. 41, no. 2, pp. 205-217, Mar. 2010, doi: 10.1007/s00158-009-0419-8.

[35] S. T. Buckland, B. Efron, and R. J. Tibshirani, "An Introduction to the Bootstrap.," Biometrics, vol. 50, no. 3, p. 890,1994 , doi: 10.2307/2532810.

[36] C. Spearman, "The Abilities of Man their Nature and Measurement," Nature, vol. 120 , no. 3014 , pp. 181183, 1927, doi: 10.1038/120181a0.

[37] B. Mondal, "Artificial intelligence: state of the art," in Intelligent Systems Reference Library, vol. 172, 2019, pp. 389-425.

[38] R. J. Sternberg, "Intelligence: State of the art," Dialogues Clin. Neurosci., vol. 14, no. 1, pp. 19-27, 2012.

[39] D. Brodić and A. Amelio, "Artificial Intelligence and Turing Test," 2020, pp. 1-5.

[40] M. Fox, M. Ghallab, G. Infantes, and D. Long, "Robot introspection through learned hidden Markov models," Artif. Intell., vol. 170, no. 2, pp. 59-113, Feb. 2006, doi: 10.1016/j.artint.2005.05.007.

[41] G. C. e R. R. Y. Bernadette BouchonMeunier, Ed., "Modern Information Processing". Elsevier, 2006.

[42] A. Simões, J. M. Viegas, J. T. Farinha, and I. Fonseca, "The State of the Art of Hidden Markov Models for Predictive Maintenance of Diesel Engines," Qual. Reliab. Eng. Int., vol. 33, no. 8, pp. 2765-2779, Dec. 2017, doi: 10.1002 /qre.2130.

[43] A. Marjanovic, G. Kvascev, P. Tadic, and Z. Djurovic, "Applications of predictive maintenance techniques in industrial systems," Serbian J. Electr. Eng., vol. 8, no. 3, pp. 263-279, 2011, doi: 10.2298/SJEE1103263M. 
[44] F. A. P. de Figueiredo, "Inteligência Artificial e Machine Learning: Introdução," no. March, 2020, doi: 10.13140/RG.2.2.10108.90241.

[45] M. Pelikan, D. Goldberg, and K. Sastry, "Bayesian Optimization Algorithm, Decision Graphs, and Occam's Razor," 2000. Accessed: Jun. 04, 2020. [Online]. Available: http://medallab.org/files/2000020.pdf.

[46] R. R. F. Attux and F. J. Von Zuben, “Árvores de Decisão," p. 22, 2010.

[47] J. R. Quinlan, "Induction of decision trees," Mach. Learn., vol. 1, no. 1, pp. 81-106, 1986, doi: $10.1007 / \mathrm{bf00116251.}$

[48] L. Breiman, J. Friedman, C. J. Stone, and R. A. Olshen, "Classification and Regression Trees". 1984.

[49] J. R. Quinlan, "C4.5: programs for machine learning". San Francisco, CA, USA.: Morgan Kaufmann Publishers Inc., 1993.

[50] Y. Amit and D. Geman, "Shape Quantization and Recognition with Randomized Trees," Neural Comput., vol. 9, no. 7, pp. 1545-1588, Oct. 1997 ,

doi: 10.1162/neco.1997.9.7.1545

[51] G. Biau, "Analysis of a random forests model," J. Mach. Learn. Res., vol. 13, pp. 1063-1095, 2012.

[52] L. Breiman, "Random Forests," Mach. Learn., vol. 45, no. 1, pp. 5-32, 2001, doi:

10.1017/CBO9781107415324.004.

[53] N. Amruthnath and T. Gupta, "Factor Analysis in Fault Diagnostics Using Random Forest," Ind Eng Manag., vol. 8, no. 1, pp. 1-10, 2019, doi: 10.4172/2169-0316.1000278.

[54] A. Liaw and M. Wiener, "Classification and Regression by randomForest," $R$ News, vol. 2, no. 3, pp. 18-22, 2002.

[55] V. S. Dave and K. Dutta, "Neural network based models for software effort estimation: A review," Artif. Intell. Rev., vol. 42, no. 2, pp. 295-
307, 2014, doi: 10.1007/s10462-0129339-x.

[56] J. Rodrigues, I. Cost, J. T. Farinha, M. Mendes, and L. Margalho, "Predicting motor oil condition using artificial neural networks and principal component analysis," Eksploat. $i$ Niezawodn. - Maint. Reliab., vol. 22, no. 3, pp. 440-448, Jun. 2020, doi: 10.17531/ein.2020.3.6.

[57] J. A. Rodrigues, J. T. Farinha, M. Mendes, I. Gonçalves, and L. Margalho, "Modelling Diesel Engine Oil Condition Using Artificial Neural," emaintance2019, 2019. https://www.researchgate.net/publicat ion/333176391_Modelling Diesel E ngine_Oil_Condition_Using_Artificia 1_Neural (accessed May 16, 2020).

[58] Haibo He and E. A. Garcia, "Learning from Imbalanced Data," IEEE Trans. Knowl. Data Eng., vol. 21, no. 9, pp. 1263-1284, Sep. 2009, doi: 10.1109/TKDE.2008.239.

[59] R. Lippmann, "An introduction to computing with neural nets," IEEE ASSP Mag., vol. 4, no. 2, pp. 4-22, 1987 , doi: 10.1109/MASSP.1987.1165576.

[60] S. Titri, H. Boumeridja, D. Lazib, and N. Izeboudjen, "A reuse oriented design methodology for artificial neural networks implementation," in Twelfth Annual IEEE International ASIC/SOC Conference (Cat. No.99TH8454), 1999, pp. 409-413, doi: 10.1109/ASIC.1999.806544.

[61] H. Rahmanifard and T. Plaksina, "Application of artificial intelligence techniques in the petroleum industry: a review," Artif. Intell. Rev., vol. 52, no. 4, pp. 2295-2318, Dec. 2019, doi: 10.1007/s10462-018-9612-8.

[62] O. Altıntaş, M. Aksoy, E. Ünal, O. Akgöl, and M. Karaaslan, "Artificial neural network approach for locomotive maintenance by monitoring dielectric properties of engine lubricant," Measurement: Journal of the International 
Measurement Confederation, vol. 145. pp. 678-686, 2019, doi: 10.1016/j.measurement.2019.05.087.

[63] T. Hongxiang, L. Yuntao, and W. Xiangjun, "Application of Neural Network to Diesel Engine SOA," in 2011 Third International Conference on Measuring Technology and Mechatronics Automation, Jan. 2011, pp. 555-558, doi: 10.1109/ICMTMA.2011.141.

[64] H.-C. Kim, S. Pang, H.-M. Je, D. Kim, and S. Yang Bang, "Constructing support vector machine ensemble," Pattern Recognit., vol. 36, no. 12, pp. 2757-2767, Dec. 2003, doi: 10.1016/S0031-3203(03)00175-4.

[65] K. Salahshoor, M. Kordestani, and M. S. Khoshro, "Fault detection and diagnosis of an industrial steam turbine using fusion of SVM (support vector machine) and ANFIS (adaptive neuro-fuzzy inference system) classifiers," Energy, vol. 35, no. 12, pp. 5472-5482, Dec. 2010, doi: 10.1016/j.energy.2010.06.001.
Creative Commons Attribution License 4.0 (Attribution 4.0 International, CC BY 4.0)

This article is published under the terms of the Creative Commons Attribution License 4.0

https://creativecommons.org/licenses/by/4.0/deed.en_US 